\title{
Novel diamond shuttle to deliver flexible neural probe with reduced tissue compression
}

\author{
Kyounghwan Na', Zachariah J. Sperry ${ }^{2,3}$, Jiaao Lu', Mihaly Vöröslakos ${ }^{1,4}$, Saman S. Parizi ${ }^{1}$, Tim M. Bruns ${ }^{2,3}$, \\ Euisik Yoon ${ }^{1,5,6}$ and John P. Seymour ${ }^{1,7,8}$
}

\begin{abstract}
The ability to deliver flexible biosensors through the toughest membranes of the central and peripheral nervous system is an important challenge in neuroscience and neural engineering. Bioelectronic devices implanted through dura mater and thick epineurium would ideally create minimal compression and acute damage as they reach the neurons of interest. We demonstrate that a three-dimensional diamond shuttle can be easily made with a vertical support to deliver ultra-compliant polymer microelectrodes (4.5- $\mu \mathrm{m}$ thick) through dura mater and thick epineurium. The diamond shuttle has $54 \%$ less cross-sectional area than an equivalently stiff silicon shuttle, which we simulated will result in a 37\% reduction in blood vessel damage. We also discovered that higher frequency oscillation of the shuttle $(200 \mathrm{~Hz})$ significantly reduced tissue compression regardless of the insertion speed, while slow speeds also

independently reduced tissue compression. Insertion and recording performance are demonstrated in rat and feline models, but the large design space of these tools are suitable for research in a variety of animal models and nervous system targets.
\end{abstract}

\section{Introduction}

Implantable sensor arrays, especially when deployed in neural mapping studies, are most accurate and insightful when damage and disruption to nervous system circuitry is minimized. Previous work demonstrated that size of an implant effects the neuron count and several glia markers of reactivity ${ }^{1,2}$. A precise function correlating implant size to tissue damage is still missing, but evidence suggests that care should be taken to minimize the damage to blood vessels which densely permeate nervous tissue ${ }^{3-6}$. Central nervous system (CNS) neurons, for example, are located within $\sim 15 \mu \mathrm{m}$ of a capillary vessel ${ }^{7-9}$ so damage to the blood-brain barrier is unavoidable. When designing an implantable device, the penetrating structure must be stiff enough to reach the desired target with accuracy and,

Correspondence: John P. Seymour (john.p.seymour@uth.tmc.edu)

'Electrical Engineering \& Computer Science Department, University of Michigan, Ann Arbor, MI 48105, USA

2Biomedical Engineering Department, University of Michigan, Ann Arbor, Ml 48105, USA

Full list of author information is available at the end of the article more challenging, piercing a tough fibrous outer membrane protecting the neurons. These challenges have opposing solutions-make the device as fine as possible for minimal bleeding and cellular disruption, yet ensure it is sufficiently stiff so as not to buckle or move off-target during penetration. The challenge is underscored when the covering membrane, the epineurium or dura mater in the peripheral or central nervous systems, respectively, are left intact during insertion.

Epineurium and dura mater are made of collagen fibers interspersed with elastin and fibroblasts that are at once tough and flexible ${ }^{10,11}$. Cranial and spinal dura mater have a Young's modulus in the range of $2-100 \mathrm{MPa}^{12}$ (depending on species and location) and so compared to the parenchyma, whose modulus is $\sim 5 \mathrm{kPa}$. They provide the primary resistance to device insertion. In the feline dorsal root ganglia (DRG), the epineurial thickness is in the range of $20-100$ micrometers (typically $80 \mu \mathrm{m})^{13}$. The dura can often be separated with minimal damage to the parenchyma with surgical training and skill, however, the creation of dural flaps severs blood vessels extending

\section{(c) The Author(s) 2020}

(c) Open Access This article is licensed under a Creative Commons Attribution 4.0 International License, which permits use, sharing, adaptation, distribution and reproduction cc) in any medium or format, as long as you give appropriate credit to the original author(s) and the source, provide a link to the Creative Commons license, and indicate if changes were made. The images or other third party material in this article are included in the article's Creative Commons license, unless indicated otherwise in a credit line to the material. If material is not included in the article's Creative Commons license and your intended use is not permitted by statutory regulation or exceeds the permitted use, you will need to obtain permission directly from the copyright holder. To view a copy of this license, visit http://creativecommons.org/licenses/by/4.0/. 
into brain and allows the brain to swell and dehydrate ${ }^{4}$. Furthermore, surgically removing the epineurium at the DRG or anywhere in the periphery is not done in practice without damaging the nervous tissue. As such, a minimally invasive solution to penetrating through CNS and peripheral nervous system (PNS) membranes is needed.

State-of-the-art sensor arrays have taken different approaches to the problem of penetration when the membrane cannot be removed. One high-density and popular electrophysiology research tool is the "Utah" array ${ }^{14,15}$, which is a silicon array comprised of many $\sim 70 \mu \mathrm{m}$ diameter needles. It is inserted with a pneumatic insertion tool and can achieve an insertion velocity of $12 \mathrm{~m} / \mathrm{s}^{16}$. The array has a wide base so it naturally stops when fully inserted but also may induce significant compression or impact trauma. No published quantification of the insertion force and compression distance exists, but significant bleeding has been reported ${ }^{17,18}$. Potentially related to this issue, the histology clearly shows encapsulation around the shank tips ${ }^{17,19,20}$ with a typical electrode-tip to axon distance of $40-150 \mu \mathrm{m}$. The resulting tissue restructuring around these microelectrodes prevents high-fidelity chronic recording, although it is still effective in recording population-based action potentials and for electrical stimulation ${ }^{21}$. Another popular recording/stimulation array, but with a smaller footprint and greater electrode density, are planar silicon probes. Historically these have been called "Michigan" arrays but these micromachined silicon structures are now made by many groups ${ }^{22-26}$ and companies. They are typically inserted in the CNS using a manual manipulator at a moderate speed so as to monitor for buckling during insertion. Given the risk of buckling or fracture, silicon planar probes are rarely used to penetrate tough membranes and there is even less known about the insertion mechanics. One study also demonstrated there is a chronic breach in the blood-brain-barrier for rigid intracortical structures ${ }^{27}$.

New research has been focused on implanting flexible arrays with assistance from a variety of techniques. Given the impressive flexibility and stretchability of nerves, ganglia, and the spinal cord, researchers have attempted to insert flexible forms of microelectrodes. The most common insertion techniques are pulling the array through the tissue using thread and needle, a dissolvable stiffener, or a removable shuttle, otherwise known as a stylet. Several groups have demonstrated the pull-through technique for intrafascicular recording used a fine commercial needle and suture ${ }^{28,29}$. The diameters of these suture needles are hundreds of microns and risk causing significant trauma. As Wurth et. al. carefully demonstrated in their histology the electrode to axon distance is typically 100 microns or more over time ${ }^{29}$. A dissolvable stiffener comes at the cost of greater volume given the temporary material is several orders of magnitude less stiff than many inorganics ${ }^{30,31}$, let alone the potential reactivity induced by the degraded polymer itself. The shuttle or stylet approach ${ }^{2,32-35}$ allows for stiffer materials and thus a smaller footprint than commercial needles or organic stiffeners.

\section{Our approach}

This paper seeks to address the combined insertion challenge of minimizing the cross-sectional area and the tissue compression. This work adds to previous research using a shuttle approach but having a unique T-shaped geometry and material to form a three-dimensional ultrananocrystalline diamond (UNCD) shuttle with 13fold greater stiffness than a similar cross-section made with conventional silicon (Fig. 1a). UNCD has the highest modulus of elasticity $(800-1000 \mathrm{GPa})^{36,37}$ of any conformal thin-film and $5 \mathrm{X}$ and $3 \mathrm{X}$ higher than silicon ${ }^{34,35}$ and carbon $^{32}$. We demonstrate a simple 2-mask method of fabrication (Fig. 1b, c). We also describe a biological model of blood vessel damage that others can use to predict damage from any arbitrary geometry. We also quantify the insertion dynamics in phantom and various tissues. Finally, we use the shuttle to demonstrate the delivery of and neural recordings from an ultra-compliant polyimide array (Fig. 1d). We expect this new ability to deliver higher-density bioelectronics with less compression and less vascular damage will improve the understanding of CNS and PNS function.

\section{Results \\ Blood vessel damage model}

While a reduction of area potentially leads to diminution of insertion damage to tissue, we have no biological evidence that this rule is still valid for the T-shaped geometry. To address this, we modeled the damage using an available model of the density, size, and length of micro-vascular segments in the brain ${ }^{8,9}$. A threedimensional micro-vascular network data for rat brain is publicly available (https://physiology.arizona.edu/people/ secomb/network/brain99). We overlaid the contour of a variety of devices onto the microvasculature maps provided initially by scanning electron microscope (SEM) imaging and 3D reconstruction. The model assumes that the device contour damages any vessel for which there is contact. This approach allows us to assess the performance of T-shape geometry in terms of a quantitative analysis of blood vessel invasion. The numbers of microvascular segments cut by the footprints of the shanks were counted throughout 1000 insertion iterations with randomized angles and locations for two equivalently rigid geometries (Fig. 2). The chosen T-beam cross-section contour was $11 \times 65 \mu \mathrm{m}$ on the planar portion and a vertical support that was $27.5-\mu \mathrm{m}$ deep that tapered from 

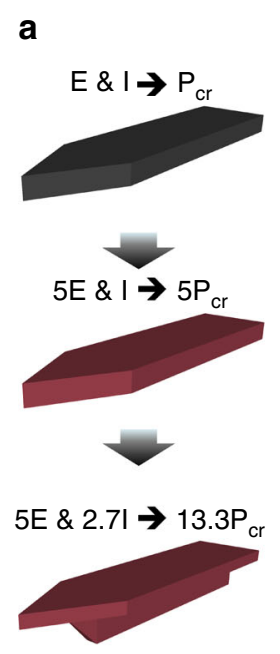
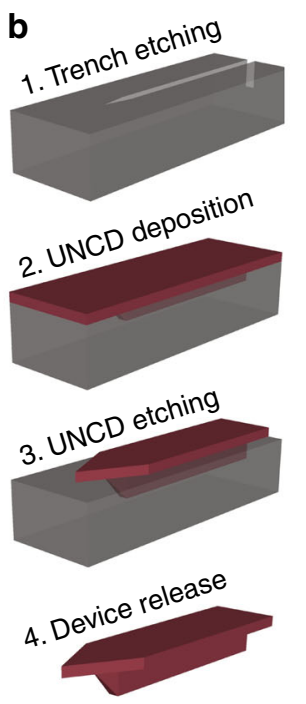

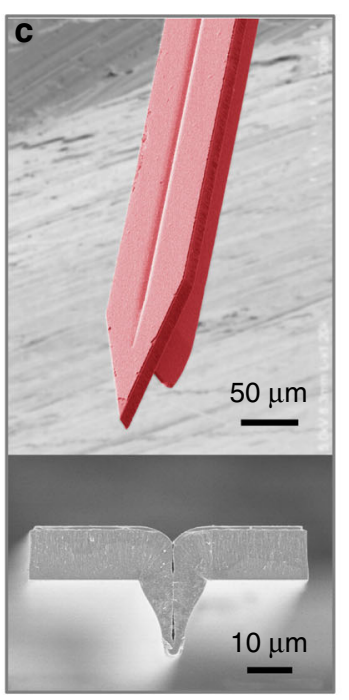

d

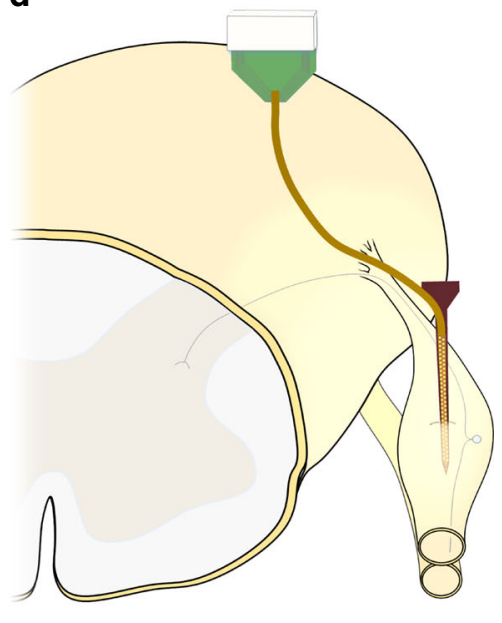

Fig. 1 Insertion concept, design concept, and fabrication of a ultra-nano-crystalline diamond (UNCD) shuttle. a Progression of design improvement from a simple silicon shuttle, with a buckling load of $P_{c r}$ to an improvement of $13.3^{*} P_{c r}$ by changing the Young's Modulus, $E$, and moment of inertia, I. b Process flow of UNCD shuttle requiring only two masks (not shown) at steps 1 and 3. c SEM of released UNCD shuttle. d Insertion of flexible electrode array into a dorsal root ganglia (DRG) using a rigid shuttle and retraction

16 to $2-\mu \mathrm{m}$ in width. This T-shaped UNCD (T-UNCD) was used for this model and throughout the study. The silicon device that has an equivalent buckling strength is $34-\mu \mathrm{m}$ thick. All devices in this study are $65-\mu \mathrm{m}$ wide which is generally the accepted width of a minimally damaging brain probe for chronic recording ${ }^{25,38}$. On average the simulation produced a $36.6 \%$ reduction in blood vessel damage caused by the T-UNCD relative to the silicon probe $34-\mu \mathrm{m}$ thick (Si-34) rectangular silicon device. Two common points of reference are a $15-\mu \mathrm{m}$ thick silicon probe, which is sufficient for insertion in rodent models with the dura removed, and another commercially available neural probe that is $50-\mu \mathrm{m}$ thick and intended for larger animal models. A T-UNCD structure (T-UNCD SMALL) that has an equivalent stiffness as a silicon probe $15-\mu \mathrm{m}$ thick (Si-15) and considerably smaller area is theorized with even structures 8$\mu \mathrm{m}$ wide actually fabricated (Supplementary Fig. 1). The simulation results were 1.4 vessels damaged by T-UNCD SMALL compared to 5.8 vessels damaged by $\mathrm{Si}-15$. By contrast, the $65 \times 50-\mu \mathrm{m}$ geometry caused damage that was 9.7-fold greater than the smaller T-UNCD.

\section{Creating 3-D UNCD structures}

The diamond T-beam shape was successfully created by patterning a tapered trench into silicon and then filling it with a moderately conformal UNCD diamond. The conformality of UNCD deposition is related to the sticking coefficient and varies in a large range according to the deposition condition and the species involved ${ }^{39}$. Our specific conditions and sticking coefficient were unknown because the hot filament chemical vapor deposition method used for this work was provided by an outside vendor and the details were proprietary. We developed a method to model the deposition profile and estimate the sticking coefficient during deposition. This was critical for defining the proper silicon trench profile (Fig. 1c) and thus minimize void creation in the shuttle's vertical support. Our deposition model (Supplementary Note 1) fit best $\left(R^{2}>0.94\right)$ with a sticking coefficient, $\eta_{0}=0.079$. The void formed was less than $0.4 \mu \mathrm{m}$ across because of the trench's taper angle of $70^{\circ}$. The angle of the silicon trench can be modified for deeper or wider geometries in the future by combining different doses of isotropic and anisotropic dry etching.

\section{Buckling load calculations and measurements}

The most dominant mechanism of mechanical failure for insertion of a needle-shaped device is due to buckling. An insertion will end up with a failure when the buckling load is lower than the required insertion force. In this case, the shank keeps deflecting more without a load increase and breaks eventually when the deflectioninduced stress exceeds the fracture strength of the material. Buckling loads for 15- $\mu \mathrm{m}$ thick planar $\mathrm{Si}(\mathrm{Si}-15)$, $50-\mu \mathrm{m}$ thick planar $\mathrm{Si}$ (Si-50), 11- $\mu \mathrm{m}$ thick planar UNCD (P-UNCD) and T-shaped UNCD (T-UNCD) shanks were measured (Fig. 3a). The characterization of buckling load was essential to analyze the results of insertion testing. Another goal of the buckling load measurement is the validation of the elastic modulus of $\mathrm{UNCD}$ and its structural design. The buckling loads of Si-15 and Si-50 


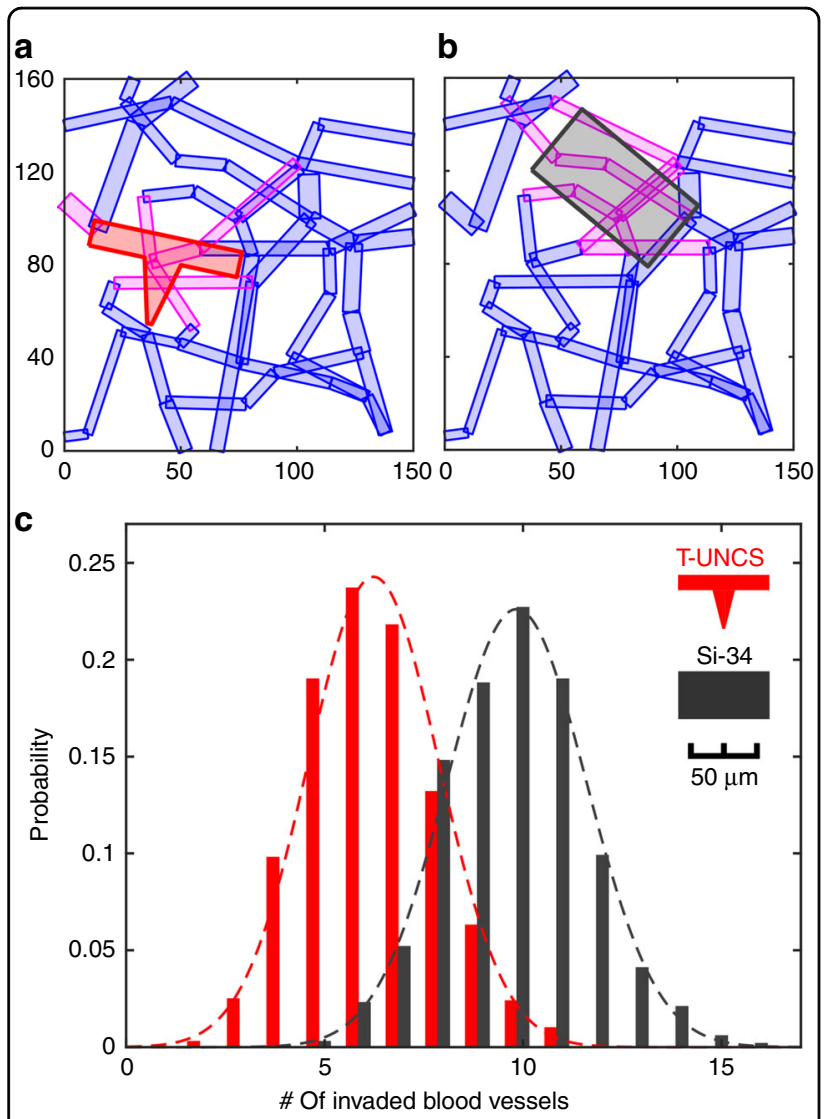

Fig. 2 Simulation result of blood vessel invasion comparing Si (dark gray) and T-UNCD (red) having an identical buckling strength. Examples of invasion analysis with $\mathbf{a} T$ shape with $65 \mu \mathrm{m}$ by $11 \mu \mathrm{m}$ on the planar portion and $27.5 \mu \mathrm{m}$-deep, $16 \mu \mathrm{m}$ to $2 \mu \mathrm{m}$-wide trapezoid for the vertical support, which has identical buckling strength with (b). b $65 \mu \mathrm{m}$ by $34 \mu \mathrm{m}$ rectangle. c Simulation result of 1000 insertions yielded a Gaussian distribution of the number of invaded blood vessels. Mean values of two geometries from $(\mathbf{a}, \mathbf{b})$ were 6.23 and 9.84, respectively. Invaded blood vessels in $(\mathbf{a}, \mathbf{b})$ indicated in magenta. $X$ and $Y$ labels in $(\mathbf{a}, \mathbf{b})$ indicated the coordinates in $\mu \mathrm{m}$ were measured as $-8.4 \%$ and $+11.3 \%$ of the calculated value (Fig. 3a). The buckling load was calculated by Eq. (1) as:

$$
\mathrm{P}_{\mathrm{cr}}=\frac{\pi^{2} E I}{(K L)^{2}}
$$

where $E$ is the Young's modulus, $I$ is the moment of inertia, $K$ is the effective length factor, and $L$ is the length. The $K$ value is dependent on the boundary condition and here it is assumed as a fixed-pinned condition in which $K$ $=0.699$ but as discussed later this is not always true. For consistency, all shuttles were also $1.5-\mathrm{mm}$ long unless otherwise stated. The moment of inertia for a rectangular beam is simply $I=b^{*} h^{3} / 12$, where $b$ is base and $h$ is height of the rectangular beam. (See Supplementary Materials for full equation used to calculate a trapezoid-shaped Tbeam).

The variation of thickness affects the buckling load significantly because the moment of inertia is proportional to the cubic of thickness. The range of UNCD's Young's modulus has been reported as $880-1000 \mathrm{GPa}^{36,40,41}$ and our estimated value based on our known geometry best fit to $800 \mathrm{GPa}$. The measured buckling load of P-UNCD was $2.3 \%$ lower than that of the calculation, and it implies that the estimation for Young's modulus is reasonable. The measurement of T-UNCD buckling load was $15.5 \%$ lower than the calculation and this is most likely due to the imperfection of the trench filling and the remaining void.

\section{Insertion force in phantom and feline DRG}

Ex vivo tissue has a limited shelf life so many tests were conducted using a 100- $\mu \mathrm{m}$ thick PDMS sheet (Sylgard 184), to simulate dura and epinuerium, secured over a larger volume of agar gel. Our experiments showed that this phantom was stiffer than rat dura and almost as stiff
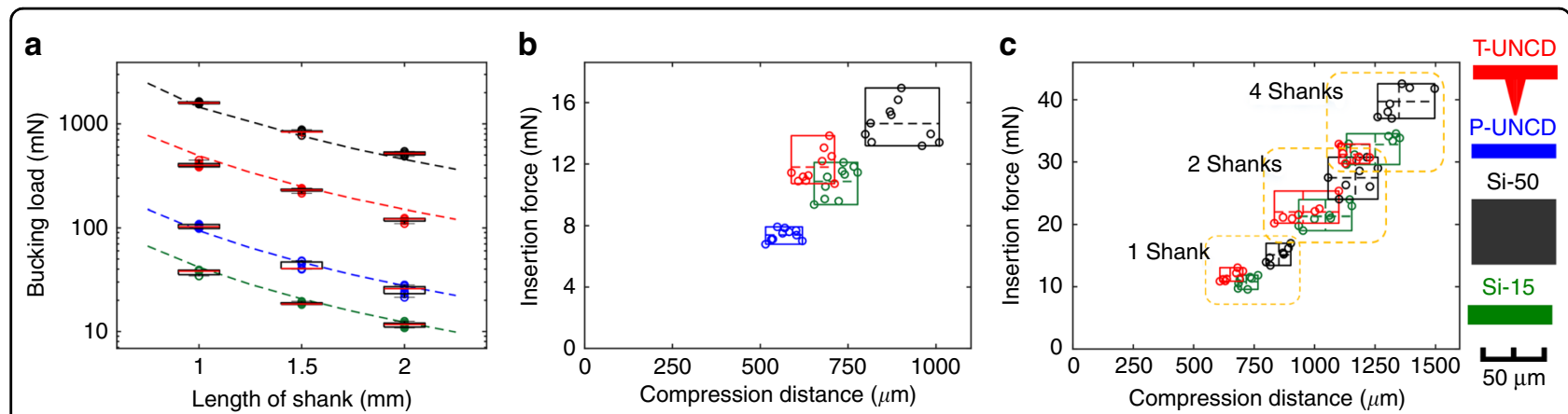

Fig. 3 Mechanical characterization of various shuttles in a tissue phantom. a Buckling load according to material, geometries and lengths, including two silicon geometries that are commercially available, $N=7$. Dashed line shows calculated value. $\mathbf{b}$ Box plot results of insertion force of single shank shuttles, $N=10$. c Box plot results of insertion force of 1, 2, and 4 shanks for three different probes, $N=7$ (planar diamond not tested). For 4-shanks, the force per shank was 7.8, 8.2, and $9.8 \mathrm{mN}$ for T-UNCD, Si-15, and Si-50, respectively. The inter-shank spacing was $250 \mu \mathrm{m}$. Insertion speed was $0.01 \mathrm{~mm} / \mathrm{s}$ in all cases 
Table 1 Average peak insertion force ${ }^{a}$

\begin{tabular}{|c|c|c|c|c|c|c|}
\hline \multirow{2}{*}{$\begin{array}{l}\text { Needle } \\
\text { Tissue }\end{array}$} & \multicolumn{4}{|l|}{ T-UNCD } & \multicolumn{2}{|c|}{ Pulled pipette $^{b}$} \\
\hline & Rat dura & Phantom & Cat DRG & Cat $^{c}$ dura & Rat dura & Phantom \\
\hline \multirow[t]{2}{*}{ Force, $\mathrm{mN}$} & $6.7 \pm 2.2$ & $12 \pm 1.0$ & $19 \pm 6.9$ & $14 \pm 2.0$ & $3.9 \pm 0.3$ & $16.7 \pm 2.0$ \\
\hline & $N=6$ & $N=10$ & $N=6$ & $N=2$ & $N=2$ & $N=8$ \\
\hline
\end{tabular}

${ }^{a}$ Oscillation force data could not be collected

b 2 - $\mu$ m diameter tip

'Lumbo-sacral region of spinal cord
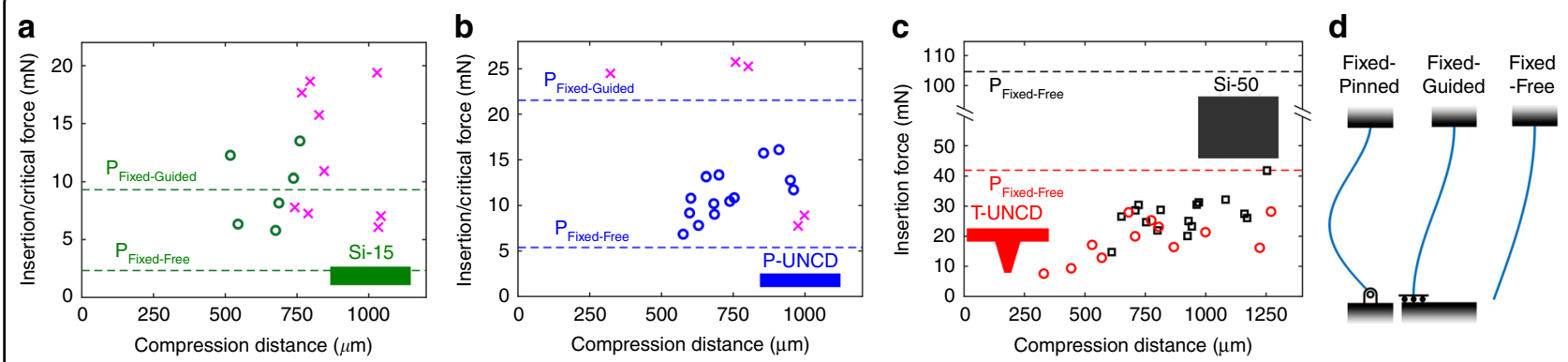

Fig. 4 Result of ex-vivo insertions into cat DRG. a 15- $\mu$ m thick Si. " $x$ " marks an insertion failure. b 11- $\mu$ m thick planar UNCD. " $x$ " marks an insertion

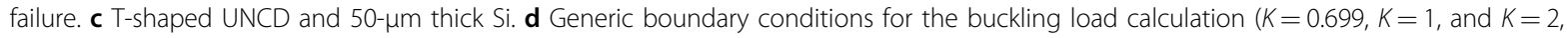

respectively). The probe backend, at top, is fixed in each scenario and tissue is at the bottom. Theoretical limits noted as dashed lines in ( $a$, $b$, $c$ )

as feline DRG epineurium (Table 1). Sylgard 184 $(1-2 \mathrm{MPa})^{42}$ and agar have similar moduli as dura ${ }^{12}$ and parenchyma $^{43,44}$, respectively. Other groups have modeled brain as agar ${ }^{45-47}$ but this is not expected to model an intact epineurium or dura mater. The chisel-shaped tips for all variations of shuttles had identical angles $\left(14^{\circ}\right.$ half angle) and the thickness varied based on film thickness, which was chosen because it is the standard tip of all Michigan-style probes.

A typical force response is an increasing force during the initial insertion until an abrupt drop indicates a puncture event. This force-distance pattern of one or more punctures in soft-tissue is well-documented ${ }^{48}$. It was expected that the thinner tip required less insertion force than thicker geometries as was actually observed (Fig. 3b), though it was not linear. T-UNCD consistently showed multiple puncture events indicated by two force peaks. This corresponds to the tips of the horizontal part and the vertical part of $\mathrm{T}$-shape, respectively (Supplementary Fig. 3b). The first peak force occurred at the range of P-UNCD insertion forces $(7.4 \mathrm{mN})$ and the second occurred following a further increase of force. The average peak insertion force for this T-UNCD was $11.8 \mathrm{mN}$ and was $8.7 \%$ higher for the $\mathrm{Si}-15$ shuttle (Fig. $3 \mathrm{~b}$ ).

The insertion force of 1,2 , and 4 shanks for each cohort were compared to investigate the behavior of multi-shank insertion (Fig. 3c). Multiple shanks were $250 \mu \mathrm{m}$ center- to-center from an adjacent shank. The force versus compression results, regardless of material and geometry, were less than a linear sum. Similarly, a three-dimensional assembly of six 4-shank UNCD arrays resulted in a decreasing force when normalized per shank (Supplementary Fig. 4).

Ex vivo insertion tests with explanted feline L6, L7, and S1 DRG were conducted to evaluate the feasibility of epineurium penetration. The average insertion forces required for successful penetration of P-UNCD, Si-15, TUNCD, and Si-50 were 11.3 $\pm 2.7,9.4 \pm 3.1,18.8 \pm 6.8$, and $27.1 \pm 6.0 \mathrm{mN}$, respectively (Fig. 4). However, Si-15 had the most failures ( 9 failures in 15 trials) followed by PUNCD (4 failures in 19 trials), with no failures observed for the other two geometries. Our results indicate that the T-UNCD shuttle will penetrate the feline epineurium with $100 \%$ success like the $\mathrm{Si}-50$ variant but with only about half of the cross-sectional area. P-UNCD and Si-15 will not reliably penetrate. In a qualitative in vivo test, the same T-UNCD design penetrated feline spinal cord (lumbar-sacral levels) nine times with zero failures where the dura is expected to be even thicker.

\section{Mechanical dynamics of insertion: speed and oscillation}

In an effort to better understand the effect of linear speed and high-speed oscillation we modified our previous force sensor experiments. A piezoelectric motor 


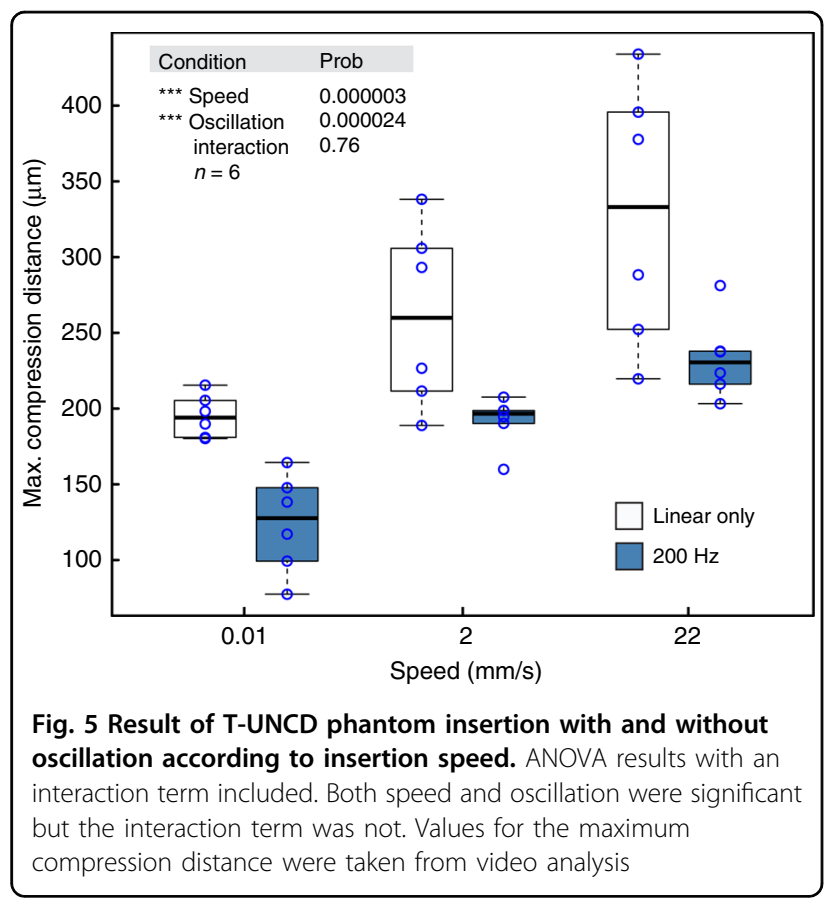

capable of uniaxial movement with an amplitude of up to $70 \mu \mathrm{m}$ was mounted onto a linear motor. A macro lens and video camera were also added to collect highresolution compression information (Supplementary Fig. $3 c)$. Both linear speed and oscillation frequency were varied and the maximum compression distance (taken from annotated video images) was measured for each condition $(N=6$; Fig. 5$)$. We found the greatest compression was $328 \mu \mathrm{m}$ and occurred at $22 \mathrm{~mm} / \mathrm{s}$, linear motion only. At the other extreme, the compression was $124 \mu \mathrm{m}$ at $0.01 \mathrm{~mm} / \mathrm{s}$ and an oscillation of $200 \mathrm{~Hz}$. This represents a relative reduction of $62 \%$. A graph of position and velocity over time for several tested motor parameters are provided (Supplementary Fig. 6). A side-by-side video comparison of the UNCD shuttle at $0.01 \mathrm{~mm} / \mathrm{s}$ with and without oscillation is also provided (Supplementary Video 1).

Oscillation frequencies of 10 and $100 \mathrm{~Hz}$ were tested at $2 \mathrm{~mm} / \mathrm{s}$ and a similar trend was observed with increasing frequency, which lowered the magnitude and variance of the compression ( $p=0.087$, Supplementary Fig. 5). We also achieved a significant reduction of compression with a pipette (2- $\mu \mathrm{m}$ tip diameter) with $200 \mathrm{~Hz}$ oscillation ( $p=$ 0.0007, Supplementary Fig. 7).

\section{In vivo demonstration in feline DRG and spinal cord}

The T-UNCD shuttle was inserted into the S1 or S2 DRG of three separate felines at $1.3 \mathrm{~mm} / \mathrm{s}$ without oscillation. One animal had mechanical testing performed, which also included successful shuttle penetrations through the spinal cord dura (Table 1). In the other two animals, functional arrays were mounted on the T-UNCD shuttles (Fig. 6) and successfully recorded neural activity. Evoked activity was recorded from a variety of stimulus types across the span of the array, including cutaneous neurons from unique locations in the perineal region and base of the tail (Fig. 7). Overall, baseline RMS of our measured noise was $14.7 \pm 3.1 \mathrm{uV}$ and SNR was $6.1 \pm 3.8$ (SNR calculation described in methods). In the first experiment, a total of 28 different channels had a unit out of 42 functional channels, spanning $1 \mathrm{~mm}$ in depth across the array. We observed 7 putative units on subsets of these 28 channels, which were overlapping due to the closeness of the electrodes (40- $\mu \mathrm{m}$ pitch). In the second animal experiment, we observed approximately 40 putative units out of 48 channels spanning $1.06 \mathrm{~mm}$ in depth when the probe was in a fixed position. When the same probe was retracted, neural activity was observed on all 59 functional channels, including one putative bladder afferent (not shown). As described further in Supplementary Note 3, in the second experiment we observed amplitudes greater than $1 \mathrm{mV}$ and the median peak-topeak amplitude for all experimental trials was $91 \mu \mathrm{V}$.

\section{In vivo demonstration in rat brain}

We inserted the T-UNCD shuttle into the motor cortex through dura mater at $0.01 \mathrm{~mm} / \mathrm{s}$ with and without oscillation $(N=6)$ without fracture or failure. The depth of insertion was $1.5 \mathrm{~mm}$. Without oscillation, the compression was $642 \mu \mathrm{m}$, and with $200 \mathrm{~Hz}$ oscillation the compression was $488 \mu \mathrm{m}$, a reduction of $24 \%(p=0.25$, $t$-test, two-sided). Separately, we also inserted the T-UNCD-polymer device through dura mater of anesthetized rats ( 2 insertions in each of 2 rats) also without failure. The flexible electrode was attached to the T$\mathrm{UNCD}$ in the same way as in the feline surgery. After successful insertion the probe was separated from the UNCD shuttle (Supplementary Video 2) and wideband $(0.3 \mathrm{~Hz}-3000 \mathrm{~Hz})$ and single unit neuronal activity were recorded from the somatosensory cortex of the rat $(N=1$, Supplementary Fig. 9a, b). The spontaneous neuronal activity was recorded for 30 minutes and 20 putative single units were found (Supplementary Fig. 9). The baseline RMS of our measured noise was $8.23 \pm 1.72$ and the SNR was $21.11 \pm 7.04$.

\section{Discussion}

We demonstrated that in rat and feline models of dura and thick epineurium, our novel T-beam UNCD shuttle can deliver a thin, flexible high-density microelectrode array that would be especially useful in neural mapping studies. The reduced-footprint with high stiffness features of the shuttle exhibited better insertion features in tissue analogues and ex vivo neural tissue than standard probes. There are also many design opportunities for thicker or thinner UNCD shuttles whose thickness, including the 

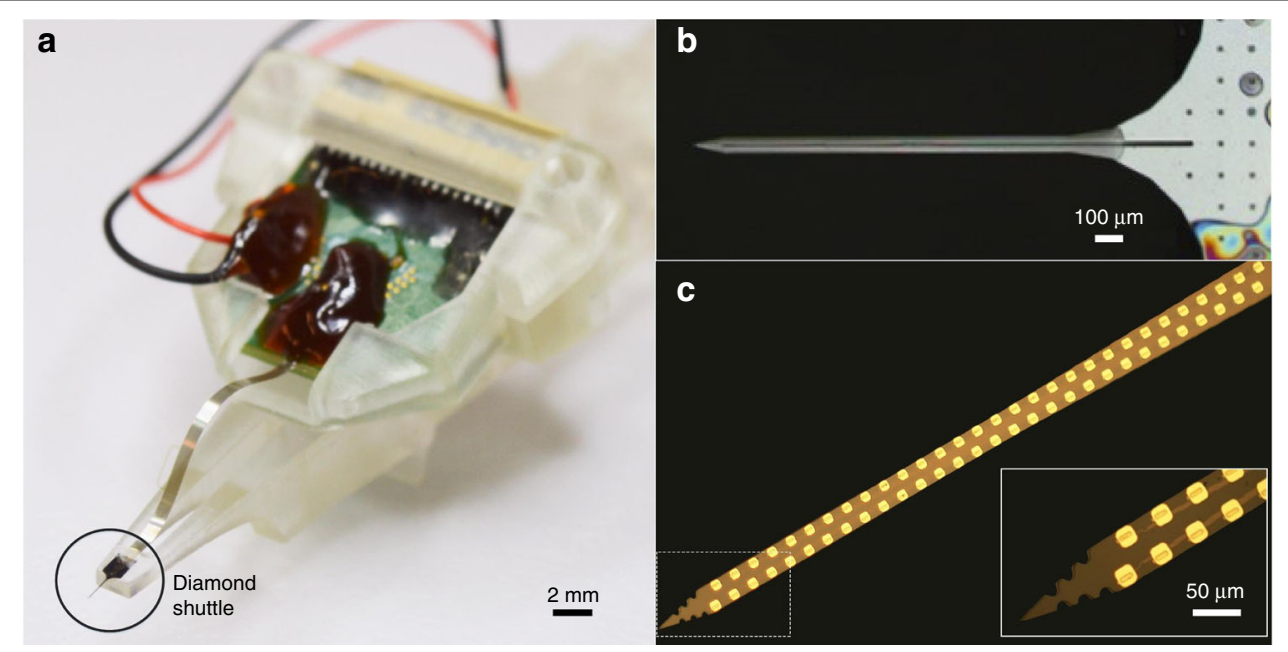

Fig. 6 Assembly of high density flexible array mounted on UNCD shuttle. a Photograph of entire assembly including the flexible polyimide array mounted on a UNCD shuttle. PCB is secured by a removable jacket that slides in a track of the shuttle jig. The insertion motor (not shown) is connected to the 3D printed shuttle jig. b Top view of PEG-coated UNCD shuttle with coating 1-2 $\mu$ m thick. c Polyimide-based flexible array with 60 recording sites and (inset) zoomed view of the array tip

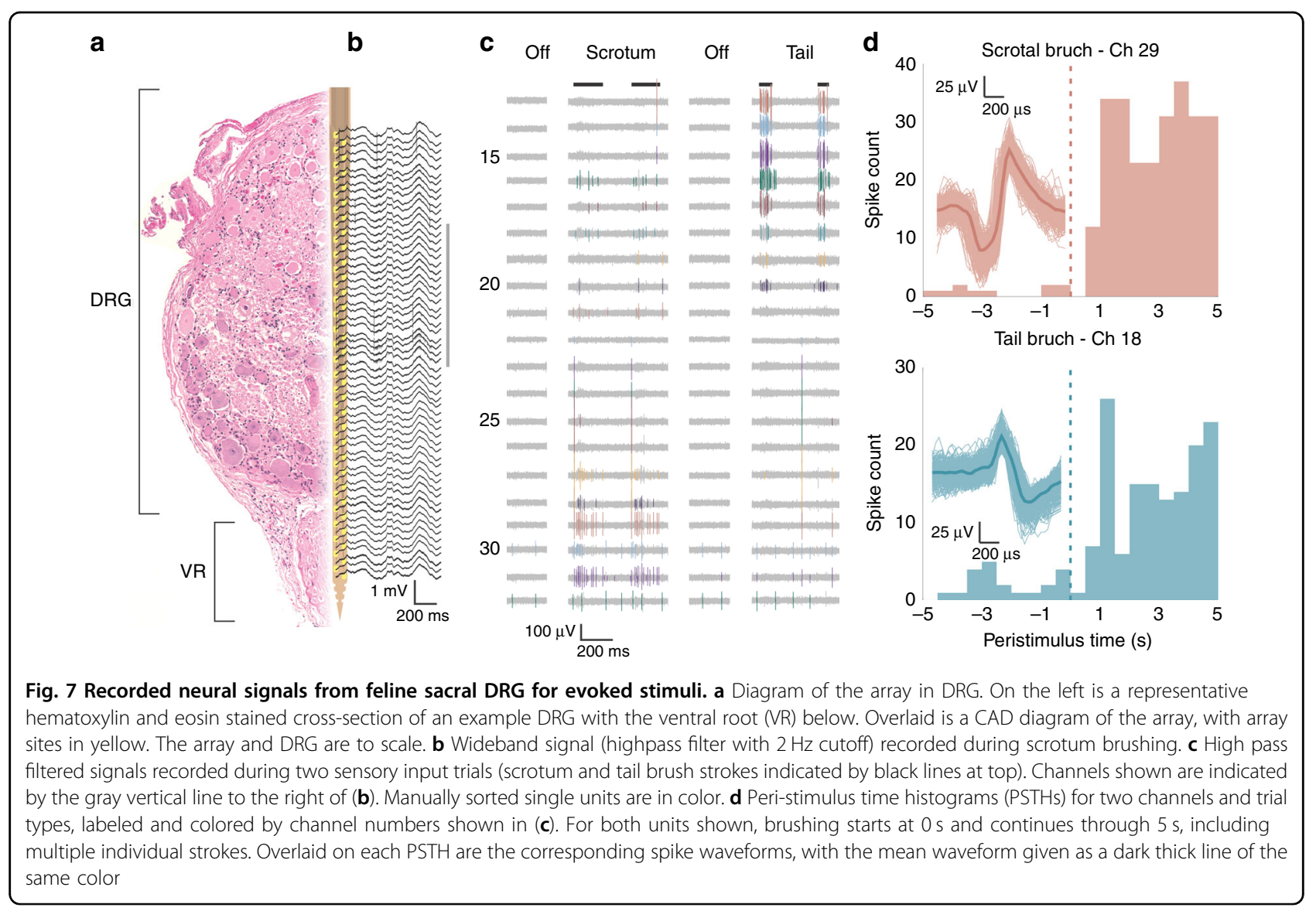

vertical support, can be in the range of $5-52 \mu \mathrm{m}$, which is potentially translated into the range of buckling load up to $1001 \mathrm{mN}$. As we demonstrate in our results, important considerations like the boundary condition of a buckling model are especially important for nerves and ganglia in the periphery. We also compare this shuttle approach to others. 
Buckling model highly dependent on boundary conditions

Throughout the ex vivo insertion tests with explanted feline DRG, many trials for Si-15 and P-UNCD resulted in failures (Fig. 4a, b) even though this was not expected based on the standard assumption of a probe tip "pinned" to stationary tissue (Fig. 4d). This commonly cited condition, where $K=0.699$, may work for rodent brain implants but was incorrect for our ex vivo DRG experiments. Our results implied that the boundary condition at the contact between the tip and the tissue varied across trials presumably due to a varying angle of contact, the shape and curvature of the targeting tissue, and the friction of the tip at the surface. Also, that is a probable situation for in vivo insertion into peripheral nerve which will have similar variations. Adding to that risk is the breathing artifact observed in larger animal models. In these situations, the boundary condition is expected to be somewhere between fixed-guided (where the tip is pinned sufficiently to the tissue but the tissue itself rotates or moves; example failure in Supplementary Video 3) or fixed-free (where the tip does not remain embedded in the surface and free to slip). This latter case is most likely due to a large insertion angle, which is challenging to avoid on highly curved surfaces. Also, a duller tip will not pin as effectively as a sharp one. The ex vivo insertion results suggested that it would be safe and wise to design the mechanics of a shuttle based on the worst-case boundary condition, especially when inserted into the PNS. This means the structure should be $8 \mathrm{X}$ stiffer than one designed for the fixed-pinned condition.

\section{Advantages of T-UNCD over other shuttle approaches}

Mounting a polymer array on the shuttle and the separation after insertion is an important, practical issue for a successful in vivo experiment. Fortunately, a planar polymer array will self-align on a planar shuttle due to geometrical similarity and surface tension when dipped in a solution of water or polyethylene glycol. This may be a simpler approach than using a cylinder-shaped shuttle, e.g. tungsten wire or carbon wire ${ }^{32,33}$ where the fabrication is expensive and does not scale to multi-shank devices. Also the resulting cross-section is a rectangle tangent to a circle and thus creating more damage than either alone. T-UNCD has the advantages of both stiffness and a matching geometry for the high-density polymer arrays used here and by other groups ${ }^{28,29,35}$. Also, compared to the stylet approach, the planar shuttle is more appropriate to deliver multi-shank electrodes readily enabling higher channel count. Furthermore the TUNCD forms a sharp tip that is effectively notched to create a micropost. Luan et al. ${ }^{32}$ demonstrated the micropost but this version required focused ion beam etching which is slow and costly compared to large-scale microfabrication. Nonetheless, the micropost concept is advantageous if the immediate release of a device is critical in an application and the potential of bending the polymer tip is not a concern. Future work may use the T$\mathrm{UNCD}$ as a micropost in order to speed the release after insertion in feline DRG experiments where breathing movement was significant. In the above acute recording demonstration, the shuttle remained for almost an hour at a time and in each case the shuttle was broken from breathing movements. When the shuttle was removed within $5 \mathrm{~min}$ of implant, the shuttle was intact, but releasing the biosensor array within 5 min using polyethylene glycol was not possible in our experience. Thus the micropost approach with the UNCD would be ideal for chronic recordings in large animals with significant tissue movement. Combined with robotics, as demonstrated in reference ${ }^{49}$, even a single stiff micropost could be used to quickly insert many flexible tines at once.

We believe that T-UNCD is superior to other shuttles even when a large insertion force is not required (e.g., when dura mater is removed). Assuming $6 \mathrm{~mm}$ length of a shank, buckling loads of T-UNCD SMALL and Si-15 are estimated as $1.9 \mathrm{mN}$ and $1.6 \mathrm{mN}$, and the former theoretically reduces the blood vessel damage to $24 \%$ of the latter (Supplementary Fig. 2). T-UNCD SMALL has 37\% less cross-sectional area than $20 \mu \mathrm{m}$-diameter tungsten wire which has an estimated buckling load of $1.8 \mathrm{mN}$.

One limitation of UNCD for applications where there is a lot of movement is the brittleness of the material compared to most metals like stainless steel. For comparison, the fracture toughness of silicon (commonly used shuttle) versus UNCD versus SS316 is $0.7-0.95^{50}, 4.7-7.2$, and $112-278 \mathrm{MPa} \mathrm{m}^{1 / 2}$, respectively. While silicon and UNCD will fracture before yielding, their fracture strength is 1.0 and $5.2^{36} \mathrm{GPa}$, respectively. SS316 begins to yield at only $290 \mathrm{MPa}$. As we discussed, an application like the feline spinal cord or DRG means great care must be taken to isolate the breathing movements. UNCD is more robust than silicon, but significantly less fracture tolerant than most metals.

\section{Insertion dynamics with oscillation}

The tissue compression in some scenarios was clearly visible, especially in feline ex vivo DRG samples where the small size of the ganglion underscored the surface movement (Fig. 4 and Supplementary Video 3). Previous work in rats, albeit without an intact dura, demonstrated that low speed reduced force and tissue compression at a speed as low as $0.2 \mathrm{~mm} / \mathrm{s}^{51}$. Therefore, our evident tissue compression at a much lower speed of $0.01 \mathrm{~mm} / \mathrm{s}$ prompted further investigation beyond speed. The ability to reduce insertion force and compression with oscillations has been documented in mosquitos ${ }^{52}$ and demonstrated with a small needle attached to a vibratory actuator with a kilohertz frequency ${ }^{53}$. In the former case, 
slow oscillations of up to $15 \mathrm{~Hz}$ using a three-part cutting system make a fascinating but complicated micromachine. In the latter case, the oscillations were $600 \mu \mathrm{m}$ in amplitude and lateral to the direction of insertion. While this reduced both compression and the insertion force, this risks damage to the nervous tissue. To address this, we employed a piezo stage with motion only in the direction of penetration and tested for the first time the combination of speed and frequency.

To study this required an accurate measure of compression. Initially, the experiments only collected force versus position data with low resolution video recording. In those experiments, we could not measure the maximum compression distance but only the total distance the probe moved before the peak force was observed. This over-estimates the compression distance since slow or small rupture events occurred before a larger rupture event, as others have reported ${ }^{51,54}$. Later we added video recording and a stabilized $4 \mathrm{X}$ macro lens (Supplementary Fig. 3c). From this the maximum compression (Fig. 5 and Supplementary Fig. 5) could be more accurately measured with annotated video images and on-screen measurements.

For some time, some neuroscientists have used slowspeed insertion for its perceived reduction in dimpling and tissue $\operatorname{strain}^{55}$. Our data confirm that slow speed, independent from oscillations, reduced the compression distance even in the context of a tougher membrane. Interestingly, high-frequency oscillation creates higher speed but in this case also significantly reduced compression and variability. The kinematics of the movement is straightforward to model (Supplementary Fig. 6) and the maximum instantaneous speed and acceleration is calculated in Supplementary Table 1. Given that the components of the insertion force includes the compression force (non-linear spring model), friction, and cutting forces ${ }^{48}$, a more careful study should be conducted to isolate the effect of speed and oscillation on each. Further research should also measure the acute damage to the blood-brain-barrier ${ }^{4,27,46}$ over this range of motion. Until more is known about the effect of oscillation on the vascular system, the best strategy would be to simply turn off oscillation after the initial membrane rupture event is detected.

\section{Materials and methods \\ Blood-vessel damage model}

A publicly available three-dimensional micro-vascular network data for rat brain (https://physiology.arizona. edu/people/secomb/network/brain99 $)^{8,9}$ was used to simulate the blood vessel invasion induced by the insertions of various geometries. For simplicity, the 3-D model was projected into a 2-D plane assuming that the insertion was vertical and straight. 1000 insertion simulations were conducted per geometry and the inserted geometry was randomly located in terms of $\mathrm{x}$, $\mathrm{y}$ locations and rotation per trial. A segment of blood vessel was classified as damaged when a device crossed the entire width of a segment. The distribution of the number of damaged blood vessels was fit to a Gaussian distribution to extract the mean value and the deviation. MATLAB code for blood vessel damage is available from the authors on request.

\section{UNCD fabrication}

The process flow is illustrated in Fig. 1c. First, standard photolithography defined a trench pattern on a 4" silicon wafer (first mask). A tapered trench was formed on silicon wafer by the combination of DRIE and isotropic dry etching. Smooth sidewalls were achieved by thermal oxidation and removal followed by another thermal oxidation for sacrificial layer growth. Afterward, 11- $\mu$ m thick UNCD film was deposited by hot filament chemical vapor deposition (CVD) at Advanced Diamond Technologies, Inc. (Romeoville, IL). Then, 2- $\mu \mathrm{m}$ thick plasma-enhanced CVD silicon dioxide was deposited at $350^{\circ} \mathrm{C}$ to be used as a hard mask for UNCD etching (second and final mask). Following photolithography, silicon dioxide and UNCD were dry-etched sequentially using $\mathrm{C}_{4} \mathrm{~F}_{8}$ and $\mathrm{O}_{2}$ chemistries, respectively. The UNCD-only devices were released in buffered hydrofluoric acid by soaking for $6 \mathrm{~h}$ and carefully rinsed with deionized water. A deposition model of UNCD in a trench is described in Supplementary Note 1.

\section{Mechanical characterization}

The inital setup for mechanical characterization was built using a precision load cell (Model 31, Honeywell, $490 \mathrm{mN}$ maximum), a digital multimeter (34011A, Agilent) and a motorized translation stage (MTS50-Z8, Thorlabs). This setup was used for data collected in Figs. 3, 4, and Supplementary Fig. 4 and illustrated in Supplementary Fig. 3a. For the oscillation and speed studies (Fig. 5, Supplementary Figs. 5 and 7) we used a piezostage HS1.70 from Piezoconcept (Lyon, France) mounted on the linear motor. The piezomotor was driven by a function generator where a $10 \mathrm{~V}$ sine input produced a $70-\mu \mathrm{m}$ PP amplitude. The silicon or UNCD shuttle was fixed to the end of load cell and the voltage output of the load cell was recorded through the ADC (225 samples per sec.) while the stage transfers the counterpart. The exerted force and travel distance were calculated by conversion of recorded data. The velocity and acceleration of the linear motor were $0.01 \mathrm{~mm} / \mathrm{s}$ and $0.1 \mathrm{~mm} / \mathrm{s}^{2}$, respectively.

A tissue phantom was formed by a $100-\mu \mathrm{m}$ thick Sylgard 184 sheet secured over $0.7 \%$ agar gel. The ex vivo DRG and spinal cord tissues were used within $72 \mathrm{~h}$ of extraction from felines used for other research purposes. All samples were kept in saline and continually rehydrated 
during an experiment to minimize mechanical degeneration or stiffening of tissues.

The recorded videos and the force-distance plots were used for the failure criteria of ex vivo DRG insertion. An insertion trial was classified as a failure when buckling was obvious from the video. In such cases, it was also evident in the force plot as there was no rupture event, i.e. drop in force over time. In other cases the shuttle fractured due to large bending.

\section{Maximum compression distance measurement}

To capture more precise compression data during oscillation we used a Canon ES Rebel 5 with a macro lens (MP-E Macro $65 \mathrm{~mm} \mathrm{1:2.8)} \mathrm{set} \mathrm{to} \mathrm{4X} \mathrm{zoom} \mathrm{mounted} \mathrm{on}$ an optical bench (Supplementary Fig. 3c). The insertion process was recorded over the full length of penetration, $1.5 \mathrm{~mm}$. These videos were then analyzed in Photoshop. Initially, a line marking the point of contact was annotated over the video, then a second line was added at the point of maximum compression. A pixel to pixel measurement was made in Photoshop and converted to distance. The pixel to distance ratio used in these videos was identical for all and determined by the known width of the shuttle $(65 \mu \mathrm{m})$. The angle between the camera lens and phantom surface was measured (within 1 degree) and used to correct for the final maximum compression value; the average angle was 7 degrees.

\section{Polymer array fabrication}

A flexible electrode array was fabricated broadly as we and others have described ${ }^{33,56}$. One layer of 2.2- $\mu \mathrm{m}$ thick polyimide (PI-2610 from HD Microsystems) was spincoated on a sacrificial layer $(100 \AA \mathrm{Cr}, 500 \AA \mathrm{Au}, 500 \AA$ $\mathrm{Cr}$ ) and fully cured at $350^{\circ} \mathrm{C}$. This thin, biostable PI is BPDA-PDA $\left(3,3^{\prime}, 4,4^{\prime}\right.$-biphenyltetracarboxilic dianhydride with p-phenylene diamine $)^{57}$. This variety of polyimide was chosen because of its high yield strength, flexibility, and low water absorption ${ }^{58}$. Metal lift-off patterning was formed in several steps depending on resolution requirements. 500-nm lines of $\mathrm{Au} 450 \mathrm{~A} / \mathrm{Pt}$ 100A/Ti 50A at $60-\mathrm{nm}$ total thickness were formed using a GCA stepper i-line lithography tool. This high resolution enabled 60 traces at a maximum width of $70 \mu \mathrm{m}$ but most of the length of the array was $65 \mu \mathrm{m}$. Future work could also employ multi-layer metal. Wider and thicker metal (Au 2500A/Pt 100A/Ti 50A) was patterned using a Seuss MA-6 contact aligner over the interconnects and bond pads. $\mathrm{A} \mathrm{TiO}_{2}$ adhesion layer above and below metal traces was used to presumably improve adhesion, although not yet validated. The top PI-2010 layer was also $2.5-\mu \mathrm{m}$ thick. The PI was etched by oxygen plasma to define the shape and open the electrical contact sites. $\mathrm{Ti} / \mathrm{Au}$ electrode metal was patterned by a liftoff process and devices were released by the removal of the sacrificial layer. The electrode pitch was $40 \mu \mathrm{m}$. In order to decrease the impedance of the recording electrode sites, PEDOT-pTS was coated on $\mathrm{Au}$ electrodes as described elsewhere ${ }^{59}$. The impedance in PBS was lowered to $24.0 \pm 1.45 \mathrm{k} \Omega$ from $1.98 \pm 0.752 \mathrm{M} \Omega$ of $\mathrm{Au}$ electrode (Supplementary Fig. 8).

\section{Mounting polymer array on UNCD shuttle}

The ultra-flexible electrode array was electrically assembled on a PCB using a common ball bonding technique $^{60}$ and then mechanically assembled on a UNCD shuttle for in vivo insertion and recording. First, the PI arrays were ball bonded onto a printed circuit board ${ }^{60}$ and the assembly was placed into a 3D-printed jacket designed to slide in the track of a shuttle jig (Fig. 6a). Separately, a UNCD shuttle was aligned and mounted on a 3D-printed shuttle jig followed by dip coating into a beaker of PEG $\left(12,000 \mathrm{MW}\right.$ ) heated to $70-90^{\circ} \mathrm{C}$ (Fig. 6b). The PI array assembly was roughly aligned over the shuttle, aligning the vertical position by sliding the array assembly in its track on the jig. This position was then fixed to the shuttle backend by a small drop of PEG (4000 MW). Precise selfalignment was achieved by once again dip-coating in heated PEG (12,000 MW). The resulting film was again $1-2 \mu \mathrm{m}$ thick and transparent (Fig. 6b). This selfalignment achieved an accuracy of at least $5-\mu \mathrm{m}$.

\section{In vivo experiment, feline DRG}

Procedures were performed in three adult, intact felines (male, 4.6-6.1 kg). Neural recordings were performed in two animals while only insertion testing was performed in a third. All steps were approved by the University of Michigan Institutional Animal Care and Use Committee (IACUC; protocol PRO00007296). As described elsewhere $^{56}$, felines were sedated with a ketaminebutorphanol-dexmedetomidine intramuscular dose prior to intubation and maintenance under isoflurane anesthesia (2-4\%) with an intravenous saline drip $(5-10 \mathrm{~mL} /$ $\mathrm{kg} / \mathrm{h}$ ) and vitals monitored and maintained within normal ranges. A laminectomy was performed to expose the lumbosacral ( L7-S3) DRG. Bone screws were inserted into the iliac crests bilaterally. The feline was placed into a custom testing stand ${ }^{61}$ which supported the chest. Stainless steel wire was wrapped around each iliac crest bone screw and fixed to the stand, allowing for the lower abdomen and hind limbs to be suspended. This positioning reduced the relative motion due to breathing that would occur at the DRG. A jig holding a shuttle-mounted array was attached to a linear inserter (M-230.25, Physik Instrumente, Karlsruhe, Germany) mounted on a micromanipulator (KITE-R, World Precision Instruments, Sarasota, FL, USA), which was used to position the array just above a DRG (S1 or S2). The inserter was driven at a rate of $1.3 \mathrm{~mm} / \mathrm{s}$ to insert the array and shuttle to a depth 
of $1.4 \mathrm{~mm}$ through the DRG. A variety of sensory stimuli were applied to activate sensory neurons, including brushing of different cutaneous dermatomes related to the DRG implanted (scrotum, anus, base of tail), infusion of saline into the bladder, and skin-surface electrical stimulation. Neural signals were sampled at $30 \mathrm{kHz}$ with a Neural Interface Processor (Grapevine, Ripple, Salt Lake City, Utah, USA). After an experiment neural signals were first sorted in Offline Sorter (Plexon, Dallas, TX, USA) prior to analysis with custom scripts in MATLAB (Mathworks, Nantick, MA, USA).

\section{In vivo experiment, rat dura}

The experiment was approved by the Institutional Animal Care and Use Committee at the University of Michigan (protocol number: PRO00007275). Two male Wistar rats $(600 \mathrm{~g})$ were implanted with a UNCDpolyimide electrode under isoflurane anesthesia $(1.2-1.5 \%)$. No prior experimentation had been performed on this rat. Atropine $(0.05 \mathrm{mg} / \mathrm{kg}$, s.c.) was administered after anesthesia induction to reduce saliva production, and the rectal temperature was monitored and kept constant at $36-37^{\circ} \mathrm{C}$ with a DC temperature controller (TCAT-LV; Physitemp, Clifton, NJ, USA). Proper depth of anesthesia was maintained by confirming the lack of nociceptive reflex. A craniotomy was drilled (4 by $4 \mathrm{~mm}$ ), carefully paying attention to not damage the dura mater. The hole was cleaned and filled with sterile saline solution ( $0.9 \%$, MWI, USA) and kept wet until the custom-electrode was implanted at $1 \mathrm{~mm}$ posterior from bregma and $2.8 \mathrm{~mm}$ lateral of the midline, in the S1FL area of the somatosensory cortex.

The electrode was inserted to a $1.5 \mathrm{~mm}$ depth (shuttle was $2 \mathrm{~mm}$ long) in the cortex using the custom motor system $(0.01 \mathrm{~m} / \mathrm{s}, 200 \mathrm{~Hz}$ oscillation, Supplementary Fig. $3 c$ ). The insertion/dura penetration process was video recorded (Canon ES Rebel 5 camera was attached to a Zeiss OPMI- 1 microscope, $\times 31.25$ total magnification). In one of the rats, the separation process was initiated by applying sterile saline on the array ten minutes after insertion. Once the upper part of the polyimide was separated the UNCD shuttle was slowly moved (50 $\mu \mathrm{m}$ up and down) until a complete separation was visible under microscope. Then, the shuttle was retracted while the recording electrode was kept in place (Supplementary Video 2$)$. The recorded signals $(n=60$ channels) were amplified (200X gain) and stored after digitization at $20 \mathrm{kHz}$ sampling rate per channel (RHD2132 and RHD2000 Evaluation System, Intan Technologies, Los Angeles, CA).

The recorded data were analyzed by custom-written scripts in MATLAB (MathWorks, USA). Neuronal spikes were detected from the digitally high-pass filtered signals
$(0.5-3 \mathrm{kHz})$ by Spikedetekt2 (https://github.com/klustateam/spikedetekt2). Detected spikes were automatically sorted using KlustaKwik2 ${ }^{62}$, followed by manual adjustment of the clusters using KlustaViewa software ${ }^{63}$ to get well-isolated single units (multi-unit and noise clusters were discarded).

To determine the noise floor for each recording channel, five $100 \mathrm{~ms}$ snippets of filtered electrophysiology recording data were chosen. These snippets did not contain sorted units and did not display amplifier saturation indicative of a motion artifact. The snippets of data, best characterized as non-spiking neural activity, were then joined together in a single $500 \mathrm{~ms}$ block which was used to calculate Vrms-channel. The SNR of each sorted unit was calculated by dividing the peak-to-peak voltage of the waveform by $(3 \mathrm{x} \text { Vrms-channel })^{59}$.

All data will be available on request from authors.

\section{Outlook}

High-density neural probes for the nervous system have a long way to progress before we achieve biomimetic scale, equivalent tissue mechanics, and effectively no tissue-reactivity. Many questions remain about insertion dynamics. Several groups quantified mechanical forces as a function tip shape and diameter when penetrating into thin membranes such as pia matter ${ }^{47,54}$, but it is not clear if these relationships hold with thicker membranes. An even more basic question is how much tissue is actually cut as opposed to radially compressed for our structures or any other. Nonetheless, the modeling and materials work shown here contribute to the ongoing efforts by many. We have developed a versatile $\mathrm{T}$-shaped ultrananocrystalline diamond shuttle which provides a $56 \%$ reduction in cross-sectional area compared to a planar silicon shuttle with equivalent insertion strength, and still stiff enough to insert into different neural structures in small and medium animal models with the dura and epineurium intact. This same structure will theoretically reduce micro-vascular damage by $37 \%$ compared to standard probes. We also quantified the benefits of slow insertion and demonstrated for the first time the use of uni-directional oscillation. This oscillation significantly reduced phantom tissue compression by $31 \%$, showing the potential for limiting neural tissue impact for in vivo use. We also demonstrate one of the highest-density sensor arrays to date -60 traces and $65-\mu \mathrm{m}$ wide on a substrate only $4.5-\mu \mathrm{m}$ thick-inserted through the toughest nervous system tissues to obtain neural signals. More broadly, the use and deployment of advanced bioelectronics as developed here is critical for high resolution scientific research into the nervous system where minimal nervous system damage is needed to observe neural activity in its most native state. 


\section{Acknowledgements}

The authors thank the staff of the Lurie Nanofabrication Facility for their tool support and technical advice when needed. We thank Paras Patel for his help in PEDOT-pTS coating. We also thank Ahmad Jiman, Zhonghua Aileen Ouyang, and Lauren Zimmerman for their help in 3D printing materials for and/or data collection in feline experiments. This work was supported in part by the National Institute of Biomedical Imaging and Bioengineering of the National Institutes of Health (R21EB020811, and SPARC program Awards U18EB021760, OT2OD024907, and OT2OD023873), Kavli Foundation funding, and Seed Funding for Innovative Projects in Neuroscience from the University of Michigan Brain Initiative Working Group (MiBrain). The content is solely the responsibility of the authors and does not necessarily represent the official views of the National Institutes of Health or the University of Michigan.

\section{Author details}

${ }^{1}$ Electrical Engineering \& Computer Science Department, University of Michigan, Ann Arbor, Ml 48105, USA. ${ }^{2}$ Biomedical Engineering Department, University of Michigan, Ann Arbor, Ml 48105, USA. ${ }^{3}$ Biointerfaces Institute, University of Michigan, Ann Arbor, MI 48105, USA. ${ }^{4}$ The Neuroscience Institute, New York University, New York, NY 10016, USA. ${ }^{5}$ Center for Nanomedicine, Institute for Basic Science (IBS), Seoul 03722, Korea. ${ }^{6}$ Graduate Program of Nano Biomedical Engineering (Nano BME), Yonsei-IBS Institute, Yonsei University, Seoul 03722, Korea. 'Vivian L Smith Department of Neurosurgery, University of Texas Health Science Center, Houston, TX 77030, USA. ${ }^{8}$ Department of Electrical and Computer Engineering, Rice University, Houston, TX 77005, USA

\section{Author contributions}

K.N., J.S., Z.S., M.V., and T.B. designed experiments. K.N., J.S., Z.S., J.L., T.B., and S.P. conducted experiments. J.S., K.N., T.B., E.Y., and M.V. wrote the paper.

\section{Conflict of interest}

The authors declare that they have no conflict of interest.

Supplementary information accompanies this paper at https://doi.org/ 10.1038/s41378-020-0149-z.

Received: 11 October 2019 Revised: 21 December 2019 Accepted: 15 January 2020

Published online: 01 June 2020

\section{References}

1. Seymour, J. P. \& Kipke, D. R. Neural probe design for reduced tissue encapsulation in CNS. Biomaterials 28, 3594-3607 (2007).

2. Kozai, T. D. Y. \& Kipke, D. R. Insertion shuttle with carboxyl terminated selfassembled monolayer coatings for implanting flexible polymer neural probes in the brain. J. Neurosci. Methods 184, 199-205 (2009).

3. Salatino, J. W., Ludwig, K. A., Kozai, T. D. Y. \& Purcell, E. K. Glial responses to implanted electrodes in the brain. Nat. Biomed. Eng 1, 862-877 (2017).

4. Kozai, T. D. Y. et al. Reduction of neurovascular damage resulting from microelectrode insertion into the cerebral cortex using in vivo two-photon mapping. J. Neural Eng. 7, 46011 (2010).

5. Michelson, N. J. et al. Multi-scale, multi-modal analysis uncovers complex relationship at the brain tissue-implant neural interface: new emphasis on the biological interface. J. Neural Eng. 15, 33001 (2018).

6. Thelin, J. et al. Implant size and fixation mode strongly influence tissue reactions in the CNS. PLOS ONE 6, e16267 (2011).

7. Tsai, P. S. et al. Correlations of neuronal and microvascular densities in murine cortex revealed by direct counting and colocalization of nuclei and vessels. J. Neurosci. 29, 14553-14570 (2009).

8. Motti, E. D., Imhof, H. G. \& Yaşargil, M. G. The terminal vascular bed in the superficial cortex of the rat. An SEM study of corrosion casts. J. Neurosurg. $\mathbf{6 5}$, 834-846 (1986).

9. Secomb, T. W., Hsu, R., Beamer, N. B. \& Coull, B. M. Theoretical simulation of oxygen transport to brain by networks of microvessels: effects of oxygen supply and demand on tissue hypoxia. Microcirculation 7, 237-247 (2000).

10. Weerasuriya, A. \& Mizisin, A. P. in The Blood-Brain and Other Neural Barriers 149-173 (Springer, 2011).
11. Thomas, P. K. The connective tissue of peripheral nerve: an electron microscope study. J. Anat. 97, 35-44 (1963).

12. Maikos, J. T., Elias, R. A. I. \& Shreiber, D. I. Mechanical properties of dura mater from the rat brain and spinal cord. J. Neurotrauma 25, 38-51 (2008).

13. Ostrowski, A. K., Sperry, Z. J., Kulik, G. \& Bruns, T. M. Quantitative models of feline lumbosacral dorsal root ganglia neuronal cell density. J. Neurosci. Methods 290, 116-124 (2017).

14. Nordhausen, C. T., Rousche, P. J. \& Normann, R. A. Optimizing recording capabilities of the Utah intracortical electrode array. Brain Res. 637, 27-36 (1994).

15. Wark, H. A. C. et al. A new high-density (25 electrodes $/ \mathrm{mm} 2$ ) penetrating microelectrode array for recording and stimulating sub-millimeter neuroanatomical structures. J. Neural Eng. 10, 45003 (2013).

16. Rousche, P. J. \& Normann, R. A. A method for pneumatically inserting an array of penetrating electrodes into cortical tissue. Ann. Biomed. Eng. 20, 413-422 (1992).

17. Branner, A., Stein, R. B., Fernandez, E., Aoyagi, Y. \& Normann, R. A. Long-term stimulation and recording with a penetrating microelectrode array in cat sciatic nerve. IEEE Trans. Biomed. Eng. 51, 146-157 (2004).

18. Christensen, M. B. et al. The foreign body response to the Utah Slant Electrode Array in the cat sciatic nerve. Acta Biomater. 10, 4650-4660 (2014).

19. Wark, H. A. C., Mathews, K. S., Normann, R. A. \& Fernandez, E. Behavioral and cellular consequences of high-electrode count Utah Arrays chronically implanted in rat sciatic nerve. J. Neural Eng. 11, 046027 (2014).

20. McCreery, D., Cogan, S., Kane, S. \& Pikov, V. Correlations between histology and neuronal activity recorded by microelectrodes implanted chronically in the cerebral cortex. J. Neural Eng. 13, 36012 (2016).

21. Wendelken, S. et al. Restoration of motor control and proprioceptive and cutaneous sensation in humans with prior upper-limb amputation via multiple Utah Slanted Electrode Arrays (USEAs) implanted in residual peripheral arm nerves. J. Neuroeng. Rehabil. 14, 121 (2017).

22. BeMent, S. L., Wise, K. D., Anderson, D. J., Najafi, K. \& Drake, K. L. Solid-state electrodes for multichannel multiplexed intracortical neuronal recording. Biomed. Eng. IEEE Trans. 2, 230-241 (1986).

23. Wise, K. D. et al. Microelectrodes, microelectronics, and implantable neural microsystems. Proc. IEEE 96, 1184-1202 (2008).

24. Herwik, S. et al. Fabrication technology for silicon-based microprobe arrays used in acute and sub-chronic neural recording. J. Micromech. Microeng. 19, 074008 (2009).

25. Lopez, C. M. et al. A neural probe with up to 966 electrodes and up to 384 configurable channels in $0.13 \mu \mathrm{m}$ SOI CMOS. IEEE Trans. Biomed. Circuits Syst. 11, 510-522 (2017)

26. Cheung, K. C., Djupsund, K., Dan, Y. \& Lee, L. P. Implantable multichannel electrode array based on SOI technology. Microelectromechanical Syst. J. 12, 179-184 (2003).

27. Saxena, T. et al. The impact of chronic blood-brain barrier breach on intracortical electrode function. Biomaterials 34, 4703-4713 (2013).

28. Boretius, T. et al. A transverse intrafascicular multichannel electrode (TIME) to interface with the peripheral nerve. Biosens. Bioelectron. 26, 62-69 (2010).

29. Wurth, S. et al. Long-term usability and bio-integration of polyimide-based intra-neural stimulating electrodes. Biomaterials 122, 114-129 (2017).

30. Takeuchi, S., Suzuki, T., Mabuchi, K. \& Fujita, H. 3D flexible multichannel neural probe array. J. Micromech. Microeng. 14, 104 (2004).

31. Wu, F. et al. Silk-Backed Structural Optimization of High-Density Flexible Intracortical Neural Probes. Microelectromechanical Syst. J. 24, 62-69 (2015).

32. Luan, L. et al. Ultraflexible nanoelectronic probes form reliable, glial scar-free neural integration. Sci. Adv. 3, 1-10 (2017).

33. Boehler, C. et al. Actively controlled release of Dexamethasone from neural microelectrodes in a chronic in vivo study. Biomaterials https://doi.org/ 10.1016/j.biomaterials.2017.03.019 (2017)

34. Felix, S. H. et al. Insertion of flexible neural probes using rigid stiffeners attached with biodissolvable adhesive. J. Vis. Exp. https://doi.org/10.3791/ 50609 (2013).

35. Chung, J. E. et al. High-density, long-lating, and multi-region electrophysiological recordings using polymer electrode arrays. Neuron 101, 21-31. e5 (2018).

36. Auciello, O. \& Sumant, A. V. Status review of the science and technology of ultrananocrystalline diamond ( $\mathrm{UNCD}^{\mathrm{TM}}$ ) films and application to multifunctional devices. Diam. Relat. Mater. 19, 699-718 (2010).

37. Auciello, O. et al. Biocompatible ultrananocrystalline diamond coatings for implantable medical devices. MRS Bull. 39, 621-629 (2014). 
38. Buzsáki, G. et al. Tools for Probing Local Circuits: High-Density Silicon Probes Combined with Optogenetics. Neuron 86, 92-105 (2015).

39. Eckert, M., Neyts, E. \& Bogaerts, A. Molecular Dynamics Simulations of the Sticking and Etch Behavior of Various Growth Species of (Ultra)Nanocrystalline Diamond Films. Chem. Vap. Depos. https://doi.org/10.1002/cvde.200706657 (2008)

40. Espinosa, H. D. \& Peng, B. A new methodology to investigate fracture toughness of freestanding MEMS and advanced materials in thin film form. J. Microelectromechanical Syst. 14, 153-159 (2005).

41. Moldovan, N., Zeng, H., Carlisle, J. A. \& Divan, R. Advanced Diamond Technologies, Inc. (2010).

42. Johnston, I. D., McCluskey, D. K., Tan, C. K. L. \& Tracey, M. C. Mechanical characterization of bulk Sylgard 184 for microfluidics and microengineering. J. Micromech. Microeng. 24, 035017 (2014).

43. Subbaroyan, J., Martin, D. C. \& Kipke, D. R. A finite-element model of the mechanical effects of implantable microelectrodes in the cerebral cortex. J. Neural. Eng. 2, 103-113 (2005).

44. Gefen, A., Gefen, N., Zhu, Q., Raghupathi, R. \& Margulies, S. S. Age-dependent changes in material properties of the brain and braincase of the rat. J. Neurotrauma 20, 1163-1177 (2003).

45. Chen, Z.-J. et al. A realistic brain tissue phantom for intraparenchymal infusion studies. J. Neurosurg. 101, 314-322 (2004).

46. Bjornsson, C. S. et al. Effects of insertion conditions on tissue strain and vascular damage during neuroprosthetic device insertion. J. Neural Eng. 3, 196 (2006).

47. Obaid, A. M. et al. Ultra-sensitive measurement of brain penetration with microscale probes for brain machine interface considerations. bioRxiv Preprint at https://www.biorxiv.org/content/10.1101/454520v1 (2018).

48. Okamura, A. M., Simone, C. \& O'leary, M. D. Force modeling for needle insertion into soft tissue. IEEE Trans. Biomed. Eng. 51, 1707-1716 (2004).

49. Musk, E. An integrated brain-machine interface platform with thousands of channels. BioRXiv Preprint at https://www.biorxiv.org/content/10.1101/ 703801v2 (2019).
50. Ericson, F., Johansson, S. \& Schweitz, J. Hardness and fracture toughnessof semiconducting materials studied by indentation and erosion Techniques * Mater. Sci. Eng. A 106, 131-141 (1988).

51. Casanova, F., Carney, P. R. \& Sarntinoranont, M. In vivo evaluation of needle force and friction stress during insertion at varying insertion speed into the brain. J. Neurosci. Methods 237, 79-89 (2014).

52. Kong, X. Q. \& Wu, C. W. Mosquito proboscis: an elegant biomicroelectromechanical system. Phys. Rev. E - Stat. Nonlinear, Soft Matter Phys. 82, 1-5 (2010).

53. Yang, M. \& Zahn, J. D. Microneedle Insertion Force Reduction Using Vibratory Actuation. Biomed. Microdevices 6, 177-182 (2004).

54. Römgens, A. M., Bader, D. L., Bouwstra, J. A., Baaijens, F. P. T. \& Oomens, C. W. J. Monitoring the penetration process of single microneedles with varying tip diameters. J. Mech. Behav. Biomed. Mater. 40, 397-405 (2014).

55. Nicolelis, M. A. L. et al. Chronic, multisite, multielectrode recordings in macaque monkeys. Proc. Natl Acad. Sci. USA 100, 11041-11046 (2003).

56. Sperry, Z. J. et al. Flexible microelectrode array for interfacing with the surface of neural ganglia. J. Neural Eng. 15, 036027 (2018).

57. Rubehn, B. \& Stieglitz, T. In vitro evaluation of the long-term stability of polyimide as a material for neural implants. Biomaterials 31, 3449-3458 (2010).

58. Ordonez, J., Schuettler, M., Boehler, C., Boretius, T. \& Stieglitz, T. Thin films and microelectrode arrays for neuroprosthetics. MRS Bull. 37, 590-598 (2012).

59. Patel, P. R. et al. Chronic in vivo stability assessment of carbon fiber microelectrode arrays. J. Neural Eng. 13, 66002 (2016).

60. Stieglitz, T., Beutel, H. \& Meyer, J. U. 'Microflex' —a new assembling technique for interconnects. J. Intell. Mater. Syst. Struct. 11, 417-425 (2000).

61. Khurram, A. et al. Chronic monitoring of lower urinary tract activity via a sacral dorsal root ganglia interface. J. Neural Eng. https://doi.org/10.1088/1741-2552/ aa6801 (2017).

62. Kadir, S. N., Goodman, D. F. M. \& Harris, K. D. High-dimensional cluster analysis with the masked EM algorithm. Neural Comput. 26, 2379-2394 (2014).

63. Rossant, C. et al. Spike sorting for large, dense electrode arrays. Nat. Neurosci. 19, 634-641 (2016) 